\title{
Pregnancy Management After Cardiac Arrest in the Previous Pregnancy
}

\author{
Somer SJH*, Sinkey RG, and Louis JM
}

Department of Obstetrics and Gynecology, Division of Maternal-Fetal Medicine, University of South Florida Morsani College of Medicine, Tampa, Florida, USA

${ }^{*}$ Corresponding author: Somer SJH, Department of Obstetrics and Gynecology, Division of Maternal-Fetal Medicine, University of South Florida Morsani College of Medicine, 2 Tampa General Circle Drive Tampa, FL 33606, Florida, USA, Tel: 8132598876; E-mail: sarahsomer@health.usf.edu

Received date: Feb 20, 2017; Accepted date: Mar 07, 2017; Published date: Mar 09, 2017

Citation: Somer SJH, Sinkey RG, Louis JM. Pregnancy Management After Cardiac Arrest in the Previous Pregnancy. Gynecol Obstet Case Rep 2017, 3:1.

\section{Abstract}

Background: Little is known about the prognosis and management of pregnancies among women who experienced a cardiac arrest in a previous pregnancy.

Case study: Cardiac arrest complicated the immediate postpartum course of a multiparous patient following an otherwise uncomplicated vaginal delivery at term. Utilizing echocardiography, a saddle pulmonary embolism was diagnosed and emergent thoracotomy and embolectomy were performed as live-saving measures. After a prolonged period of rehabilitation, the patient was discharged home. She subsequently became pregnant two years later, was maintained on therapeutic anticoagulation, and delivered a viable infant at term without significant maternal or neonatal sequelae.

Conclusion: Etiology of cardiac arrest and subsequent cardiac function should direct the management of future pregnancies. Favorable pregnancy outcomes are possible among women who experienced a cardiac arrest in a previous pregnancy due to saddle emboli.

\section{Keywords: Cardiac arrest; Pregnancy; Pulmonary} embolism

\section{Introduction}

Maternal cardiac arrest is a rare and terrifying event. The incidence in the United States is estimated to be 16 per 100,000 live births [1], however, as cardiovascular disease becomes more prevalent and severe in the United States, the rate of cardiac arrest in pregnancy is thought to be increasing $[2,3]$. Maternal mortality from maternal cardiac arrest is at least $40 \%[4,5]$; if the arrest occurs prior to delivery, neonatal morbidity and mortality are high, with time from arrest-todelivery a crucial factor in survival [4].

There is a paucity of literature concerning cardiac arrest in the puerperium and most existing publications are case reports and expert opinions. The existing literature does not address long-term outcomes of survivors of cardiac arrest, and thus, counselling survivors about future reproduction outcomes is difficult. We describe a case of a patient who experienced maternal cardiac arrest, survived, and subsequently had a successful pregnancy which delivered at term.

\section{Case}

A 33-year old, African American, G3P2002 presented for scheduled induction of labor at 38 weeks and 6 days secondary to fetal growth restriction. Her pregnancy was complicated by mild intermittent asthma, obesity, and a history of gastric bypass surgery resulting in Vitamin B12 and D nutritional deficiencies. Maternal body mass index on admission was 31.1 $\mathrm{kg} / \mathrm{m}^{2}$. This multiparous patient had a routine induction course and on hospital day two had an uncomplicated vaginal delivery of a female infant weighing 2660 grams with Apgar scores of nine at both one and minutes of life. The estimated blood loss from the vaginal delivery totaled 100 milliliters $(\mathrm{mL})$. Two hours later the patient experienced an $800 \mathrm{~mL}$ postpartum hemorrhage; it was managed with uterine massage, oxytocin, and rectal misoprostol; hemoglobin 3 hours post-delivery dropped to $7.6 \mathrm{~g} / \mathrm{dL}$ from $11.4 \mathrm{~g} / \mathrm{dL}$ obtained on admission.

However, during a subsequent fundal check approximately one hour later, the patient became briefly unresponsive and was noted to be hypotensive with a blood pressure of 67/29 $\mathrm{mmHg}$. She was administered oxygen and an intravenous fluid bolus and became responsive, then reported chest tightness and light-headedness. The uterine fundus was firm with scant vaginal bleeding; an EKG noted sinus tachycardia with short PR interval and nonspecific ST wave abnormality.

After the initial episode, the patient reported that her symptoms were resolving. However, less than one hour later, her care team witnessed seizure-like activity. Diazepam was administered with no change in mental status. Her blood pressure began to drop and respirations ceased. A code blue was called and the patient was intubated; cardiac monitoring indicated pulseless electrical activity (PEA). She received epinephrine, sodium bicarbonate, and chest compressions according to advanced cardiovascular life support (ACLS) protocol, as well as two units of packed red blood cells given 
her acute anaemia. Spontaneous cardiac activity resumed for approximately five minutes followed by another episode of PEA. ACLS was again performed with return of spontaneous circulation and the patient was transferred to the intensive care unit (ICU). During transfer, she had a third episode of PEA that again responded to ACLS.

Bedside echocardiogram indicated hyperdynamic left ventricle ejection fraction; severely dilated right ventricle, severely dilated right atrium, severe tricuspid regurgitation, severe pulmonary hypertension with right ventricular systolic pressure of $75 \mathrm{mmHg}$; echodense lesion in the pulmonary artery suspicious for embolism; moderate to severely reduced right ventricle function with possible McConnell's sign; and dilated inferior vena cava. Given high suspicion for pulmonary embolism, the patient was started on a heparin drip and interventional radiology was consulted. Thrombolytics could not be given secondary to postpartum hemorrhage, cardiac arrest, and recent chest compressions. As such, cardiothoracic surgery was consulted and recommended emergent pulmonary thrombectomy.

Utilizing cardiopulmonary bypass, the patient underwent an embolectomy of an extensive saddle pulmonary embolism (Figure 1) and subsequent placement of an inferior vena cava (IVC) filter; her chest wound was left open and packed. She became coagulopathic intraoperatively, resulting in 2,000 mL uterine blood loss which stabilized with Bakri balloon placement, oxytocin, and misoprostol.

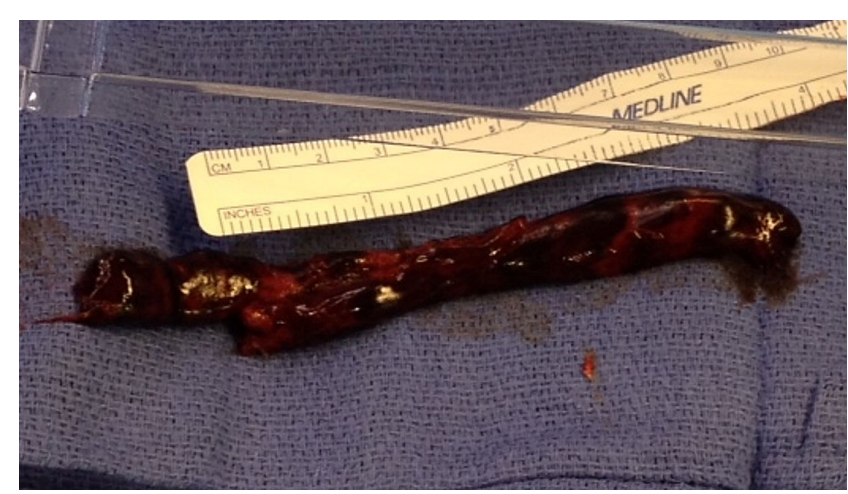

Figure 1 Saddle pulmonary embolism, this photograph depicts the saddle pulmonary embolus removed after an uncomplicated vaginal delivery followed by cardiac arrest. An emergent echocardiogram showed a hypoechoic area in the pulmonary artery and emergent thoracotomy was performed to remove the saddle pulmonary embolism.

On postpartum day one her hemoglobin was 8.5 and coagulation studies were within normal limits; by that time the patient had received 12 units of PRBCs, 10 units of fresh frozen plasma, seven packs of platelets, 40 units of cryoprecipitate, factor seven, and desmopressin. She had significant neurological symptoms, including weakness and myoclonus, and was diagnosed with probable ischemic stroke. On postpartum day two her chest wound was closed and the Bakri balloon was removed; she was extubated on postpartum day five; she was discharged to the inpatient rehabilitation center on warfarin on postpartum day 21.

The patient was readmitted to the hospital numerous times over the next year for a variety of complaints including seizures, chest pain, and dizziness. Thrombophilia workup, consisting of proteins $\mathrm{C}$ and $\mathrm{S}$, prothrombin, lupus anticoagulant, factor $\mathrm{V}$ Leiden, Factor 2, cardiolipin, and beta-2 glycoprotein, was negative; no further cardiac or thrombotic events were diagnosed. A normal echocardiogram was reported two months after discharge. Her neurological symptoms largely resolved and her seizures were well controlled on Keppra.

During this patient's prolonged postpartum course the rounding obstetric team discussed contraception several times at length. Due to the uncertainty of the maternal state should she conceive, and with pregnancy as the only known provoking event for thrombus formation, both the patient and her spouse were advised against future pregnancy.

Despite advice against future pregnancy, approximately two years later the patient was unexpectedly found to be pregnant. Due to the uncertainty of the maternal course, and the possibility of worsening maternal condition or death, the patient was counselled about the range of potential outcomes and management options. The patient declined termination of pregnancy. Of note, the patient had few memories from her previous delivery and postpartum course due to the anoxic brain injury. Her spouse, however, interpreted the team's contraception advice as "she would never be able to get pregnant again."

Since the thrombophilia workup was negative and pregnancy was the only identifiable and modifiable known risk factor for her previous event, she was treated with therapeutic anticoagulation throughout her pregnancy. The patient did have short-term memory loss and organized her medications and upcoming appointments in a notebook that she kept with her at all times. She was admitted six times during the pregnancy for a variety of complaints including chest pain, lower extremity edema, and shortness of breath. In total, she had 4 echocardiograms; the most concerning findings on transthoracic echocardiography at 27 weeks indicated pulmonary hypertension with a pulmonary artery pressure of $44 \mathrm{mmHg}$ to $49 \mathrm{mmHg}$. However, cardiac catheterization at 31 weeks revealed normal pulmonary artery pressures of 23, 11 and $17 \mathrm{mmHg}$. In total, this patient was inpatient for 15 days prior to delivery.

A scheduled induction of labor occurred at 37 weeks gestational age. Due to a persistent category II tracing unresponsive to resuscitative measures, the patient underwent a cesarean delivery with bilateral tubal ligation. The female infant weighed $2810 \mathrm{~g}$ and had Apgars of five, eight, and nine. The patient was kept on telemetry throughout the admission with no abnormal findings. She did well postoperatively and was discharged home on post-op day four. For the next six weeks of the postpartum period both the mother and infant remained well without complication. 


\section{Comment}

Although rare, maternal cardiac arrest has implications so dire that it warrants attention and preparation. Long-term outcomes of survivors of cardiac arrest in the general population are addressed in the literature. However, not much is known about long-term outcomes of patients who experience cardiac arrest during pregnancy. This case demonstrates the following key points about cardiac arrest in pregnancy: successful management of subsequent pregnancies is possible, but long-term morbidities may still be present.

One long-term morbidity essential to discuss is the potential for neurologic deficits. Neurologic deficits among cardiac arrest survivors are well described [6,7]. However, these deficits in an obstetric population require a multi-disciplinary approach to adequately treat. For instance, patients may not have the capacity to understand common instructions, such as birth control directions. Therefore, options that do not require patient compliance may better serve this population. In this case, long-acting reversible contraception arranged prior to hospital discharge could have prevented an unintended pregnancy.

It is also notable that the patient had high health care utilization in her subsequent pregnancy. There were multiple hospitalizations and procedures performed due to her symptoms; factors that may impact healthcare utilization include the etiology of the cardiac arrest, cardiac recovery and presence of comorbid conditions. These factors should be evaluated with counselling of these women regarding the potential burden prior to subsequent conception.

Counseling women who have survived maternal cardiac arrest regarding future pregnancies is difficult given the paucity of literature on the topic. The etiology of the arrest is likely to influence the management and outcomes. Regardless of etiology, full cardiac rehabilitation and psychological health should be evaluated prior to another pregnancy. If repeat pregnancy occurs, termination may be offered, as it was for our patient, particularly if cardiac function is suboptimal.

This patient suffered cardiac arrest as a result of a pulmonary embolism, a risk factor for cardiac arrest, which was mitigated (although not eliminated) in her next pregnancy through anticoagulation. All women who survive maternal cardiac arrest and desire further pregnancies should be thoroughly counselled about the paucity of literature on this topic, the uncertainty regarding risk of recurrence and the potential significant burden of a subsequent pregnancy. Close observation should continue through the pregnancy, delivery, and postpartum period.

\section{References}

1. Creanga AA, Berg CJ, Syverson C, Seed K, Bruce FC, et al. (2015) Pregnancy-related mortality in the United States, 2006-2010. Obstet Gynecol 125(1): 5-12.

2. Vanden Hoek TL, Morrison LJ, Shuster M, Donnino M, Sinz E, et al. (2010) Part 12: Cardiac arrest in special situations: 2010 American Heart Association Guidelines for Cardiopulmonary Resuscitation and Emergency Cardiovascular Care. Circulation 122 (18 suppl 3): S829-S861.

3. Jeejeebhoy F, Windrim R (2014) Management of cardiac arrest in pregnancy. Best Pract Res Clin Obstet Gynaecol 28(4): 607-618.

4. Jeejeebhoy FM, Zelop CM, Lipman S, Carvalho B, Joglar J, et al. (2015) Cardiac arrest in pregnancy: AHA scientific statement. Circulation 132:1747-1773.

5. Mhyre JM, Tsen LC, Einav S, Kuklina EV, Leffert LR, et al. (2014) Cardiac arrest during hospitalization for delivery in the United States, 1998-2011. Anesthesiology120:810-818.

6. Moulaert VR, Verbunt JA, van Heugten CM, Wade DT (2009) Cognitive impairements in survivors of out-of-hospital cardiac arrest: A systematic review. Resuscitation 80: 297-305.

7. Kim YJ, Ahn S, Sohn CH, Seo DW, Lee YS, et al. (2016) Long-term neurologic outcomes in patients after out-of-hospital cardiac arrest. Resuscitation 101:1-5. 